\title{
Integral operators with the generalized sine-kernel on the real axis
}

\author{
N. A. Slavnov 1
}

\begin{abstract}
The asymptotic properties of integral operators with the generalized sine kernel acting on the real axis are studied. The formulas for the resolvent and the Fredholm determinant are obtained in the large $x$ limit. Some applications of the results obtained to the theory of integrable models are considered.
\end{abstract}

Keywords: Fredholm determinant, resolvent, asymptotic expansion

\section{Introduction}

Integral operators with a sine kernel arise in many areas of mathematical physics. The sine kernel has the form

$$
S(\lambda, \mu)=\frac{\sin \frac{x}{2}(\lambda-\mu)}{\pi(\lambda-\mu)},
$$

where $\lambda$ and $\mu$ are integration variables, $x$ is a real parameter. The operator $I+\gamma S$, where $I$ is the identity operator and $\gamma$ is a complex number, acts on an interval (or a system of intervals) $\ell$ of the real axis on functions from $L_{2}(\ell)$.

The Fredholm determinant of the integral operator $I-S$ appears in random matrix theory [1]. In the scaling limit, $\operatorname{det}_{\ell}(I-S)$ gives the probability that a matrix belonging to the Gaussian unitary ensemble has no eigenvalues in the interval $x \ell$ [2. The kernel (1.1) also appears in the

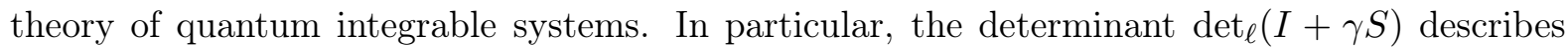
various zero temperature correlation functions of the impenetrable Bose gas model [3, 4].

In the interpretations of the sine kernel mentioned above the most interesting question is related to the large $x$ behavior of the Fredholm determinant $\operatorname{det}_{\ell}(I+\gamma S)$. This problem was studied in numerous works (see e.g. [1, 2], [5]-[12]). A very nice connection of the sine kernel to the Painlevé $\mathrm{V}$ equation was investigated in [4, 13. The methods of the asymptotic analysis

\footnotetext{
${ }^{1}$ Steklov Mathematical Institute, Moscow, Russia, nslavnov@mi.ras.ru
} 
of $\operatorname{det}_{\ell}(I+\gamma S)$ are based on the fact that at $x$ large the kernel $S$ becomes rapidly oscillating. Note however that the kernel has no saddle points, and shifts of the integration contour do not make it exponentially small. Therefore standard methods of asymptotic estimates of oscillating integrals fail in this case.

Various generalizations of the kernel (1.1) preserving its oscillating structure are known in the literature. One of such generalizations arises in the theory of truncated Wiener-Hopf operators [14, 15], where the integral operator acts on $\mathbb{R}$ and the complex number $\gamma$ is replaced by some function $\gamma(\lambda)$. Other generalizations of the sine kernel were basically used for the description of correlation functions of matrix models or quantum integrable systems equivalent to free fermions (see e.g. [16]-[24]). Most of these kernels can be presented in the form

$$
V(\lambda, \mu)=\frac{\sqrt{F(\lambda) F(\mu)}}{2 i \pi(\lambda-\mu)}\left[e_{+}(\lambda) e_{-}(\mu)-e_{-}(\lambda) e_{+}(\mu)\right]
$$

where

$$
e_{ \pm}(\lambda)=\exp \left( \pm \frac{i x p(\lambda)}{2} \pm \frac{g(\lambda)}{2}\right)
$$

and the operator $I+\gamma V$ acts on some contour $\mathcal{C}$. We call the kernel (1.2) the generalized sine kernel (GSK). The large $x$ asymptotic behavior of the Fredholm determinant $\operatorname{det}_{\mathcal{C}}(I+\gamma V)$ where studied in the works mentioned above for certain particular choices of functions $F, p$, and $g$ and the contour $\mathcal{C}$. It was shown in 25, that the Fredholm determinant asymptotic expansion depends only on the analytic properties of the functions $F, p$, and $g$ in an neighborhood of the contour $\mathcal{C}$. Knowing these properties one can evaluate the asymptotic behavior without using the explicit form of these functions.

In the present article we focus our attention on a particular case of the GSK where the integral operator $I+\gamma V$ acts on the whole real axis. We also set $p(\lambda)=\lambda$, because just this situation occurs in the most of applications. This case is more simple than the one of a finite interval (moreover a union of finite intervals). The matter is that for a finite interval $\ell$ the large $x$ asymptotic behavior of $\operatorname{det}_{\ell}(I+\gamma V)$ mostly follows from the analytic properties of the resolvent to the operator $I+\gamma V$ in vicinities of the endpoints of $\ell$. Although the methods of such analysis are now well developed (see e.g. [12, 24]), they are rather complicated technically. As a result even in the simplest cases usually it is possible to obtain explicitly only first several terms of the asymptotic expansion.

In the case of operators acting on $\mathbb{R}$ this difficulty disappears due to the absence of the endpoints. Due to this one can obtain uniform asymptotic estimates for the resolvent on the whole real axis up to exponentially small corrections. As a result it is possible to promote much further in studying of the Fredholm determinant asymptotic behavior. In some particular cases one can obtain even the complete asymptotic expansion.

Similar picture arises in the case of the GSK acting on closed contours $\mathcal{C}$. For these kernels the function $p(\lambda)$ usually is such that $\left|e^{i x p(\lambda)}\right|=1$ for $\lambda \in \mathcal{C}$. The methods of the asymptotic analysis proposed in this paper can be used in such cases as well. 
This article is organized as follows. In section 2, we announce the main results of the paper, namely, the asymptotic formula for the resolvent of the operator $I+\gamma V$ and two types of the asymptotic expansion of $\operatorname{det}_{\mathbb{R}}(I+\gamma V)$. The proofs of these results are given in sections 3 5, In section 6 we consider two applications of the results obtained.

\section{Notations and main theorems}

In this section we specify some properties of the functions $g(\lambda)$ and $F(\lambda)$ entering the kernel (1.2). Recall that we have set $p(\lambda)=\lambda$.

Let $g(\lambda)$ be holomorphic in a strip $|\Im(\lambda)|<a, a>0$. Assume also that $g(\lambda) / \lambda \rightarrow 0$ as $\Re(\lambda) \rightarrow \pm \infty$ and $|\Im(\lambda)|<a$. The function $F(\lambda)$ is holomorphic in the same strip except $n_{+}+n_{-}$simple poles in the points $r_{j}^{ \pm}$separated from the real axis. 1 . We assume that the points $r_{j}^{+}, j=1, \ldots, n_{+}$lie in the upper half-plane and $r_{k}^{-}, k=1, \ldots, n_{-}$lie in the lower half-plane (see Fig. 1). The complex number $\gamma$ is supposed to be small enough, such that zeros $s_{j}^{ \pm}$of the function $1+\gamma F(\lambda)$ are slightly shifted from the poles $r_{j}^{ \pm}$(see Fig. 1). The zeros $s_{j}^{+}, j=1, \ldots, n_{+}$ belong to the upper half-plane and $s_{k}^{-}, k=1, \ldots, n_{-}$belong to the lower half-plane. Finally we assume that $F(\lambda) \rightarrow 0$ as $\Re(\lambda) \rightarrow \pm \infty,|\Im(\lambda)|<a$ in such a way that $|\operatorname{tr} V|<\infty$.

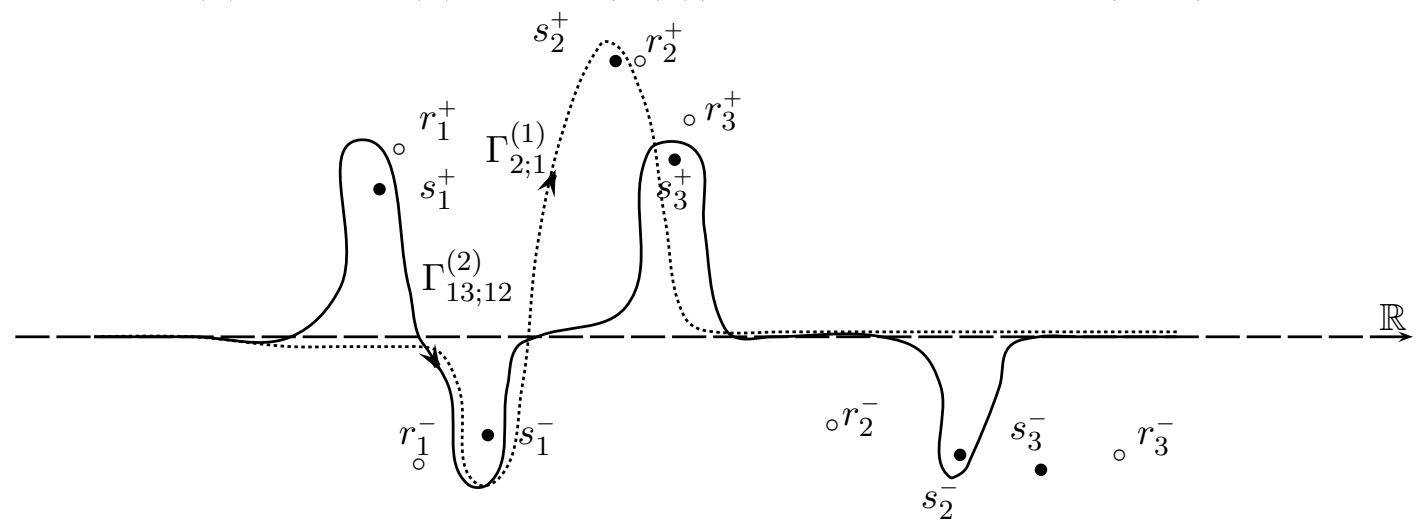

Figure 1: The poles $r_{j}^{ \pm}$are shown by $\circ$, the zeros $s_{j}^{ \pm}$of $1+\gamma F(\lambda)=0$ are shown by $\bullet$. The integration contour $\Gamma_{13 ; 12}^{(2)}$ (solid line) bypasses the points $s_{1}^{+}, s_{3}^{+}$from above and $s_{1}^{-}, s_{2}^{-}$from below. The contour $\Gamma_{2 ; 1}^{(1)}$ (dotted line) bypasses the point $s_{2}^{+}$from above and $s_{1}^{-}$from below.

Under the conditions listed above the Fredholm determinant $\operatorname{det}_{\mathbb{R}}(I+\gamma V)$ exists and it is an entire function of $\gamma$. Since at $\gamma=0$ this determinant is equal to 1 , we conclude that $\operatorname{det}_{\mathbb{R}}(I+\gamma V) \neq 0$, if $\gamma$ belongs to some vicinity of the origin. Hence, the resolvent to the operator $I+\gamma V$ exists at least for $\gamma$ small enough.

\footnotetext{
${ }^{1}$ The case of higher order poles can be analyzed by taking the limits $r_{j}^{ \pm} \rightarrow r_{k}^{ \pm}$. We do not consider this case, because the explicit formulas become much more cumbersome.
} 
Let us introduce now several functions used below. First, we define functions $\nu(\lambda)$ and $\alpha(\lambda)$ as

$$
\nu(\lambda)=\frac{-1}{2 \pi i} \log (1+\gamma F(\lambda)), \quad \alpha(\lambda) \equiv \alpha([\nu], \lambda)=\exp \left(\int_{\mathbb{R}} \frac{\nu(\mu) d \mu}{\mu-\lambda}\right) .
$$

The branch of the logarithm in (2.1) is fixed by the condition $\nu(+\infty)=0$. Clearly $\alpha(\lambda)$ has a cut on the real axis, and its limiting values $\alpha_{ \pm}(\lambda)$ from the upper and lower half-planes enjoy the property

$$
\alpha_{-}(\lambda)=\alpha_{+}(\lambda)(1+\gamma F(\lambda)), \quad \lambda \in \mathbb{R} .
$$

The limiting values $\alpha_{ \pm}(\lambda)$ can be continued to the upper (resp. to the lower) half-plane, where they are non-vanishing. At $\lambda \rightarrow \infty$ the function $\alpha(\lambda)$ behaves as

$$
\alpha(\lambda)=1+\frac{\alpha_{1}}{\lambda}+O\left(\lambda^{-2}\right), \quad \lambda \rightarrow \infty, \quad \text { where } \quad \alpha_{1}=-\int_{\mathbb{R}} \nu(\mu) d \mu .
$$

Let us also define $\left(n_{+} \times n_{-}\right)$-matrix $A^{-}$and $\left(n_{-} \times n_{+}\right)$-matrix $A^{+}$as

$$
A_{j k}^{-}=\frac{h_{k}^{-} e_{-}^{2}\left(s_{k}^{-}\right)}{s_{j}^{+}-s_{k}^{-}}, \quad A_{j k}^{+}=\frac{h_{k}^{+} e_{+}^{2}\left(s_{k}^{+}\right)}{s_{j}^{-}-s_{k}^{+}}, \quad \text { where } \quad h_{k}^{ \pm}=-\frac{\left(\alpha_{ \pm}\left(s_{k}^{ \pm}\right)\right)^{\mp 2}}{\gamma F^{\prime}\left(s_{k}^{ \pm}\right)} \text {. }
$$

We also introduce a set of contours $\Gamma_{J ; K}^{(n)}$ with $n=0,1, \ldots, \min \left(n_{+}, n_{-}\right)$. Here $J$ and $K$ are multi-indexes: $J=\left\{j_{1}, \ldots, j_{n}\right\}$ with $1 \leq j_{s} \leq n_{+}$, and $K=\left\{k_{1}, \ldots, k_{n}\right\}$ with $1 \leq k_{s} \leq n_{-}$. We set by definition $\Gamma^{(0)}=\mathbb{R}$. The contour $\Gamma_{J ; K}^{(n)}$ is a deformation of the real axis such that moving $\mathbb{R}$ to $\Gamma_{J ; K}^{(n)}$ we cross only the roots $s_{j_{1}}^{+}, \ldots, s_{j_{n}}^{+}$and $s_{k_{1}}^{-}, \ldots, s_{k_{n}}^{-}$, while other roots $s_{\ell}^{ \pm}$and all the poles $r_{\ell}^{ \pm}$should not be crossed (see Fig. 10).

Finally let

$$
\mathcal{A}_{\mathcal{C}}([g],[\nu])=-\int_{\mathcal{C}}\left(i x+g^{\prime}(\lambda)\right) \nu(\lambda) d \lambda+\int_{\mathcal{C}} \frac{\nu(\lambda) \nu(\mu)}{\left(\lambda-\mu_{+}\right)^{2}} d \lambda d \mu .
$$

Here the contour $\mathcal{C}$ is one of $\Gamma_{J ; K}^{(n)}$. The symbol $\mu_{+}$means that $\mu$ is slightly shifted to the left from the integration contour $\mathcal{C}$. Note that one can always choose the cuts of the function $\nu(\lambda)$ in such a way that the contour $\mathcal{C}$ does not cross them.

Now we are ready to formulate the main theorems on the asymptotic behavior of the resolvent and $\operatorname{det}_{\mathbb{R}}(I+\gamma V)$.

Theorem 2.1. Let $x \rightarrow \infty$ and

$$
R(\lambda, \mu)+\gamma \int_{\mathbb{R}} V(\lambda, \xi) R(\xi, \mu) d \xi=V(\lambda, \mu) .
$$

Then $R(\lambda, \mu)$ has the following form:

$$
R(\lambda, \mu)=\frac{\sqrt{F(\lambda) F(\mu)}}{2 i \pi(\lambda-\mu)}\left[f_{+}(\lambda) f_{-}(\mu)-f_{-}(\lambda) f_{+}(\mu)\right]
$$


where

$$
f_{ \pm}(\lambda)=\alpha_{\mp}^{\mp 1}(\lambda) e_{ \pm}(\lambda)\left[1+\sum_{j=1}^{n_{\mp}} \frac{D_{j}^{ \pm} h_{j}^{\mp} e_{\mp}^{2}\left(s_{j}^{\mp}\right)}{\lambda-s_{j}^{\mp}}\right]+\alpha_{ \pm}^{ \pm 1}(\lambda) e_{\mp}(\lambda) \sum_{j=1}^{n_{ \pm}} \frac{C_{j}^{ \pm} h_{j}^{ \pm} e_{ \pm}^{2}\left(s_{j}^{ \pm}\right)}{\lambda-s_{j}^{ \pm}}+O\left(e^{-a x}\right),
$$

uniformly for $\lambda \in \mathbb{R}$. The constants $C_{j}^{ \pm}$and $D_{j}^{ \pm}$can be found from the systems

$$
\left\{\begin{array} { l } 
{ C _ { j } ^ { + } - \sum _ { k = 1 } ^ { n _ { + } } A _ { j k } C _ { k } ^ { + } = 1 , } \\
{ D _ { j } ^ { + } - \sum _ { k = 1 } ^ { n _ { + } } A _ { j k } ^ { + } C _ { k } ^ { + } = 0 , }
\end{array} \quad \left\{\begin{array}{l}
C_{j}^{-}-\sum_{k=1}^{n_{-}} \tilde{A}_{j k} C_{k}^{-}=1, \\
D_{j}^{-}-\sum_{k=1}^{n_{-}} A_{j k}^{-} C_{k}^{-}=0,
\end{array}\right.\right.
$$

where $A=A^{-} A^{+}$and $\tilde{A}=A^{+} A^{-}$.

This theorem is proved in the next section.

Remark. The entries of the matrices $A^{ \pm}$are exponentially small for $x$ large enough. Therefore $\operatorname{det}(I-A) \neq 0$ and $\operatorname{det}(I-\tilde{A}) \neq 0$, and hence, each of the systems (2.9) has a unique solution.

Theorem 2.2. Let $x \rightarrow \infty$. Then the Fredholm determinant of the operator $I+\gamma V$ behaves as

$$
\operatorname{det}_{\mathbb{R}}(I+\gamma V)=e^{\mathcal{A}_{\mathbb{R}}([g],[\nu])} \operatorname{det}_{n_{+}}(I-A)\left(1+O\left(e^{-a x}\right)\right) .
$$

Here the functional $\mathcal{A}_{\mathbb{R}}([g],[\nu])$ is given by $(2.5)$ with $\mathcal{C}=\mathbb{R}$.

The proof of this theorem is given in section 4

Remark. In (2.10) the determinant of $\left(n_{+} \times n_{+}\right)$-matrix $I-A$ can be replaced by the determinant of $\left(n_{-} \times n_{-}\right)$-matrix $I-\tilde{A}$.

Theorem 2.3. Let $x \rightarrow \infty$. Then the Fredholm determinant of the operator $I+\gamma V$ behaves as

$$
\operatorname{det}_{\mathbb{R}}(I+\gamma V)=\sum_{\mathcal{C}} e^{\mathcal{A}_{\mathcal{C}}([g],[\nu])}\left(1+O\left(e^{-a x}\right)\right),
$$

where the functional $\mathcal{A}_{\mathcal{C}}([g],[\nu])$ is given by (2.5) and the sum is taken with respect to all possible contours $\mathcal{C} \in\left\{\Gamma_{J ; K}^{(n)}\right\}$ including $\Gamma^{(0)}=\mathbb{R}$.

The proof of this theorem is given in section 5 ,

\section{Asymptotic formula for the resolvent}

This section is devoted to the proof of Theorem 2.1. The operator $I+\gamma V$ belongs to the class of completely integrable operators [23, 27]. It is known [18, 23, 27] that for such operators the kernel of the resolvent has the form (2.7), where functions $f_{ \pm}(\lambda)$ solve an integral equation

$$
f_{ \pm}(\lambda)+\frac{\gamma}{2 \pi i} \int_{\mathbb{R}} \frac{e_{+}(\lambda) e_{-}(\mu)-e_{-}(\lambda) e_{+}(\mu)}{\lambda-\mu} F(\mu) f_{ \pm}(\mu) d \mu=e_{ \pm}(\lambda) .
$$


Thus, to prove Theorem 2.1 we should solve asymptotically the equation (3.1) up to $O\left(e^{-a x}\right)$ terms.

Proof of Theorem 2.1. Since for $\gamma$ small enough $\operatorname{det}_{\mathbb{R}}(I+\gamma V) \neq 0$, the solution of (3.1) exits and unique. Therefore it is enough to substitute (2.8) into the integral equation (3.1) and to check that the last one holds up to terms of order $O\left(e^{-a x}\right)$.

Consider, for instance, the equation for $f_{+}(\lambda)$. Making the substitution we find

$$
\begin{aligned}
f_{+}(\lambda)- & e_{+}(\lambda)=\frac{1}{2 \pi i} \int_{\mathbb{R}} \frac{\gamma F(\mu) \alpha_{-}^{-1}(\mu) d \mu}{\mu-\lambda+i 0}\left[1+\sum_{j=1}^{n_{-}} \frac{D_{j}^{+} h_{j}^{-} e_{-}^{2}\left(s_{j}^{-}\right)}{\mu-s_{j}^{-}}\right]\left(e_{+}(\lambda)-e_{-}(\lambda) e_{+}^{2}(\mu)\right) \\
& +\frac{1}{2 \pi i} \int_{\mathbb{R}} \frac{\gamma F(\mu) \alpha_{+}(\mu) d \mu}{\mu-\lambda-i 0} \sum_{j=1}^{n_{+}} \frac{C_{j}^{+} h_{j}^{+} e_{+}^{2}\left(s_{j}^{+}\right)}{\mu-s_{j}^{+}}\left(e_{+}(\lambda) e_{-}^{2}(\mu)-e_{-}(\lambda)\right)+O\left(e^{-a x}\right) .
\end{aligned}
$$

For convenience we have shifted $\mu-\lambda$ by $+i 0$ in the first integral and by $-i 0$ in the second one. Consider the coefficient at $e_{+}(\lambda)$. We have

$$
\begin{array}{r}
\alpha_{-}^{-1}(\lambda)\left[1+\sum_{j=1}^{n_{-}} \frac{D_{j}^{+} h_{j}^{-} e_{-}^{2}\left(s_{j}^{-}\right)}{\lambda-s_{j}^{-}}\right]-1=\frac{1}{2 \pi i} \int_{\mathbb{R}} \frac{\gamma F(\mu) \alpha_{-}^{-1}(\mu) d \mu}{\mu-\lambda+i 0}\left[1+\sum_{j=1}^{n_{-}} \frac{D_{j}^{+} h_{j}^{-} e_{-}^{2}\left(s_{j}^{-}\right)}{\mu-s_{j}^{-}}\right] \\
+\frac{1}{2 \pi i} \int_{\mathbb{R}} \frac{\gamma F(\mu) \alpha_{+}(\mu) e_{-}^{2}(\mu) d \mu}{\mu-\lambda-i 0} \sum_{j=1}^{n_{+}} \frac{C_{j}^{+} h_{j}^{+} e_{+}^{2}\left(s_{j}^{+}\right)}{\mu-s_{j}^{+}}+O\left(e^{-a x}\right) .
\end{array}
$$

The integral in the first line of (3.3) can be taken explicitly by use of $\gamma F(\lambda) \alpha_{-}^{-1}(\lambda)=\alpha_{+}^{-1}(\lambda)-$ $\alpha_{-}^{-1}(\lambda)$. Since $\alpha_{ \pm}(\lambda)$ are analytical and non-vanishing in the corresponding half-planes and due to the condition (2.3) we obtain,

$$
\begin{aligned}
& \frac{1}{2 \pi i} \int_{\mathbb{R}} \frac{\gamma F(\mu) \alpha_{-}^{-1}(\mu) d \mu}{\mu-\lambda+i 0}\left[1+\sum_{j=1}^{n_{-}} \frac{D_{j}^{+} h_{j}^{-} e_{-}^{2}\left(s_{j}^{-}\right)}{\mu-s_{j}^{-}}\right] \\
& =\frac{1}{2 \pi i} \int_{\mathbb{R}} d \mu \frac{\alpha_{+}^{-1}(\mu)-1-\left(\alpha_{-}^{-1}(\mu)-1\right)}{\mu-\lambda+i 0}+\frac{1}{2 \pi i} \int_{\mathbb{R}} d \mu \frac{\alpha_{+}^{-1}(\mu)-\alpha_{-}^{-1}(\mu)}{\mu-\lambda+i 0} \sum_{j=1}^{n_{-}} \frac{D_{j}^{+} h_{j}^{-} e_{-}^{2}\left(s_{j}^{-}\right)}{\mu-s_{j}^{-}} \\
& =\alpha_{-}^{-1}(\lambda)\left[1+\sum_{j=1}^{n_{-}} \frac{D_{j}^{+} h_{j}^{-} e_{-}^{2}\left(s_{j}^{-}\right)}{\lambda-s_{j}^{-}}\right]-1-\sum_{j=1}^{n_{-}} \frac{\alpha_{-}^{-1}\left(s_{j}^{-}\right) D_{j}^{+} h_{j}^{-} e_{-}^{2}\left(s_{j}^{-}\right)}{\lambda-s_{j}^{-}} .
\end{aligned}
$$

Thus, the equation (3.3) takes the form

$$
\sum_{j=1}^{n_{-}} \frac{\alpha_{-}^{-1}\left(s_{j}^{-}\right) D_{j}^{+} h_{j}^{-} e_{-}^{2}\left(s_{j}^{-}\right)}{\lambda-s_{j}^{-}}=\frac{1}{2 \pi i} \int_{\mathbb{R}} \frac{\gamma F(\mu) \alpha_{-}(\mu) e_{-}^{2}(\mu) d \mu}{(1+\gamma F(\mu))(\mu-\lambda-i 0)} \sum_{k=1}^{n_{+}} \frac{C_{k}^{+} h_{k}^{+} e_{+}^{2}\left(s_{k}^{+}\right)}{\mu-s_{k}^{+}}+O\left(e^{-a x}\right) .
$$


Here we have used $\alpha_{+}(\mu)=\alpha_{-}(\mu)(1+\gamma F(\mu))^{-1}$. The remaining integral can be computed asymptotically by the residues at $1+\gamma F(\mu)=0$ in the lower half-plane. Comparing then the coefficients at every $\left(\lambda-s_{j}^{-}\right)^{-1}$ we obtain

$$
D_{j}^{+}=\sum_{k=1}^{n_{+}} A_{j k}^{+} C_{k}^{+} .
$$

Similar calculation of the coefficient at $e_{-}(\lambda)$ in (3.2) leads us to equations

$$
C_{j}^{+}=1+\sum_{k=1}^{n_{-}} A_{j k}^{-} D_{k}^{+} .
$$

The equations (3.6), (3.7) yield immediately the first of the systems (2.9). The second system (2.9) follows from the analysis of the integral equation for the function $f_{-}(\lambda)$, what can be done by the same method.

\section{The first asymptotic formula for the determinant}

The leading terms of the Fredholm determinant $\operatorname{det}_{\mathbb{R}}(I+\gamma V)$ asymptotic expansion were obtained in the work [25] (see also 28] for the case $g(\lambda)=0$ )

$$
\log \operatorname{det}_{\mathbb{R}}(I+\gamma V)=\mathcal{A}_{\mathbb{R}}([g],[\nu])+o(1), \quad x \rightarrow \infty .
$$

Thus, we need only to prove that the corrections to this formula have the form of the finite size matrix determinant $\operatorname{det}(I-A)$.

Proof of Theorem 2.2. The corrections to the equation (4.1) can be computed from the following identity [18, 23]:

$$
\partial_{x} \log \operatorname{det}_{\mathbb{R}}(I+\gamma V)=\frac{\gamma}{2 \pi} \int_{\mathbb{R}} f_{+}(\lambda) e_{-}(\lambda) F(\lambda) d \lambda .
$$

Substituting here (2.8) for $f_{+}(\lambda)$ we obtain

$$
\begin{aligned}
\partial_{x} \log \operatorname{det}(I+\gamma V)=\frac{1}{2 \pi} \int_{\mathbb{R}} \gamma & F(\lambda) \alpha_{-}^{-1}(\lambda)\left[1+\sum_{j=1}^{n_{-}} \frac{D_{j}^{+} h_{j}^{-} e_{-}^{2}\left(s_{j}^{-}\right)}{\lambda-s_{j}^{-}}\right] d \lambda \\
& +\frac{1}{2 \pi} \int_{\mathbb{R}} \gamma F(\lambda) \alpha_{-}(\lambda) e_{-}^{2}(\lambda) \sum_{j=1}^{n_{+}} \frac{C_{j}^{+} h_{j}^{+} e_{+}^{2}\left(s_{j}^{+}\right)}{\lambda-s_{j}^{+}}+O\left(e^{-a x}\right) .
\end{aligned}
$$

Let us give several comments on the calculation of the integral

$$
\frac{1}{2 \pi} \int_{\mathbb{R}} \gamma F(\lambda) \alpha_{-}^{-1}(\lambda) d \lambda
$$


We have

$$
\gamma F(\lambda) \alpha_{-}^{-1}(\lambda)=\alpha_{+}^{-1}(\lambda)-\alpha_{-}^{-1}(\lambda)=\left(\alpha_{+}^{-1}(\lambda)-1+\frac{\alpha_{1}}{\lambda-\lambda_{0}}\right)-\left(\alpha_{-}^{-1}(\lambda)-1+\frac{\alpha_{1}}{\lambda-\lambda_{0}}\right),
$$

where $\lambda_{0}$ is an arbitrary complex number with positive imaginary part. Observe that due to (2.3) both terms in the r.h.s. of (4.5) behave as $O\left(\lambda^{-2}\right)$ as $\lambda \rightarrow \infty$. Therefore one can integrate each of these terms separately. The integral of the second term vanishes, since the integrand is analytical in the lower half-plane. The first term in (4.5) has only one simple pole in the upper half-plane, therefore

$$
\frac{1}{2 \pi} \int_{\mathbb{R}} \gamma F(\lambda) \alpha_{-}^{-1}(\lambda) d \lambda=\frac{1}{2 \pi} \int_{\mathbb{R}}\left(\alpha_{+}^{-1}(\lambda)-1+\frac{\alpha_{1}}{\lambda-\lambda_{0}}\right) d \lambda=i \alpha_{1} .
$$

Other integrals in (4.3) can be computed similarly to the ones considered in the previous section. Using the equations (2.9) we obtain after simple algebra

$$
\partial_{x} \log \operatorname{det}(I+\gamma V)=i \alpha_{1}-\sum_{j=1}^{n_{+}} A_{j j}^{\prime} C_{j}^{+}+O\left(e^{-a x}\right),
$$

where prime means the derivative over $x$. Using the explicit expression (2.4) for $A$ one can easily convince himself that

$$
A_{j j}^{\prime}=A_{\ell j}^{\prime}+i\left(s_{\ell}^{+}-s_{j}^{+}\right) A_{\ell j} .
$$

Then due to (2.9) we have

$$
\begin{gathered}
\partial_{x} \log \operatorname{det}(I+\gamma V)-i \alpha_{1}=-\sum_{j, \ell=1}^{n_{+}}\left[A_{\ell j}^{\prime}+i\left(s_{\ell}^{+}-s_{j}^{+}\right) A_{\ell j}\right](I-A)_{j \ell}^{-1}+O\left(e^{-a x}\right) \\
=\partial_{x} \operatorname{tr} \log (I-A)+i \sum_{j, \ell=1}^{n_{+}}\left(s_{\ell}^{+}-s_{j}^{+}\right)\left(\delta_{\ell j}-A_{\ell j}\right)(I-A)_{j \ell}^{-1}+O\left(e^{-a x}\right) \\
=\partial_{x} \log \operatorname{det}(I-A)-i \sum_{j=1}^{n_{+}} s_{j}^{+}+i \sum_{\ell=1}^{n_{+}} s_{\ell}^{+}+O\left(e^{-a x}\right)=\partial_{x} \log \operatorname{det}(I-A)+O\left(e^{-a x}\right) .
\end{gathered}
$$

Integrating over $x$ we arrive at

$$
\log \operatorname{det}(I+\gamma V)=i x \alpha_{1}+C+\log \operatorname{det}(I-A)+O\left(e^{-a x}\right),
$$

and comparing (4.10) with (4.1) we find the integration constant $C$

$$
C=-\int_{\mathbb{R}} g^{\prime}(\lambda) \nu(\lambda) d \lambda+\int_{\mathbb{R}} \frac{\nu(\lambda) \nu(\mu)}{\left(\lambda-\mu_{+}\right)^{2}} d \lambda d \mu=\left.\mathcal{A}_{\mathbb{R}}([g],[\nu])\right|_{x=0} .
$$


Thus, we have found the corrections to (4.1), and it remains to prove that the determinant of $\left(n_{+} \times n_{+}\right)$-matrix $I-A$ is equal to the determinant of $\left(n_{-} \times n_{-}\right)$-matrix $I-\tilde{A}$. For this it is enough to present $\operatorname{det}(I-A)$ as a determinant of a block-matrix of the size $\left(n_{+}+n_{-}\right) \times\left(n_{+}+n_{-}\right)$:

$$
\operatorname{det}(I-A)=\operatorname{det}\left(I-A^{-} A^{+}\right)=\operatorname{det}\left(\begin{array}{cc}
I & A^{+} \\
A^{-} & I
\end{array}\right)=\operatorname{det}\left(I-A^{+} A^{-}\right)=\operatorname{det}(I-\tilde{A}),
$$

what ends the proof.

\section{The second asymptotic formula for the determinant}

Define a set of analogs of the function $\alpha(\lambda)$ (2.1)

$$
\alpha\left(\lambda ; \Gamma_{J ; K}^{(n)}\right)=\exp \left(\int_{\Gamma_{J ; K}^{(n)}} \frac{\nu(\mu) d \mu}{\mu-\lambda}\right)
$$

where $\nu(\lambda)$ is still given by (2.1). Obviously,

$$
1+\gamma F(\lambda)=\frac{\alpha_{-}\left(\lambda ; \Gamma_{J ; K}^{(n)}\right)}{\alpha_{+}\left(\lambda ; \Gamma_{J ; K}^{(n)}\right)}, \quad \lambda \in \Gamma_{J ; K}^{(n)} .
$$

Here $\alpha_{ \pm}$are the limiting values of the function $\alpha\left(\lambda ; \Gamma_{J ; K}^{(n)}\right)$ on the contour $\Gamma_{J ; K}^{(n)}$ from the left (resp. from the right). The function $\alpha_{-}$is analytical and non-vanishing in the domain to the right from $\Gamma_{J ; K}^{(n)}$, it has zeros at $\lambda=s_{\ell}^{+}, \ell \notin J$ and poles at $\lambda=r_{\ell}^{+}, \ell=1, \ldots, n_{+}$. Similarly $\alpha_{+}^{-1}$ is analytical and non-vanishing in the domain to the left from $\Gamma_{J ; K}^{(n)}$, it has zeros at $\lambda=s_{\ell}^{-}$, $\ell \notin K$ and poles at $\lambda=r_{\ell}^{-}, \ell=1, \ldots, n_{-}$.

Lemma 5.1. Let $\nu(\lambda)$ be given by (2.1). Then

$$
\exp \left\{\mathcal{A}_{\Gamma_{J ; K}^{(n)}}([g],[\nu])-\mathcal{A}_{\Gamma_{\hat{J} ; \hat{K}}^{(n-1)}}([g],[\nu])\right\}=\frac{\alpha_{-}^{2}\left(s_{n}^{-} ; \Gamma_{\hat{J} ; \hat{K}}^{(n-1)}\right) \alpha_{+}^{-2}\left(s_{n}^{+} ; \Gamma_{\hat{J} ; \hat{K}}^{(n-1)}\right)}{\left(s_{j_{n}}^{+}-s_{k_{n}}^{-}\right)^{2} \gamma^{2} F^{\prime}\left(s_{j_{n}}^{+}\right) F^{\prime}\left(s_{k_{n}}^{-}\right)} e_{+}^{2}\left(s_{j_{n}}^{+}\right) e_{-}^{2}\left(s_{k_{n}}^{-}\right),
$$

where $\hat{J}=J \backslash j_{n}$ and $\hat{K}=K \backslash k_{n}$.

Proof. Consider the first term in (2.5) setting $\mathcal{C}=\Gamma_{J ; K}^{(n)}$ and $\mathcal{C}=\Gamma_{\hat{J} ; \hat{K}}^{(n-1)}$. The contour $\Gamma_{J ; K}^{(n)}$ is obtained from $\Gamma_{\hat{J} ; \hat{K}}^{(n-1)}$ by crossing the roots of $1+\gamma F(\lambda)$ at $s_{j_{n}}^{+}$and $s_{k_{n}}^{-}$(see Fig 2). Therefore we have

$$
-\left(\int_{\Gamma_{J ; K}^{(n)}}-\int_{\Gamma_{\hat{\jmath} ; \hat{K}}^{(n-1)}}\right)\left(i x+g^{\prime}(\lambda)\right) \nu(\lambda) d \lambda=\int_{s_{k_{n}}^{-}}^{s_{j_{n}}^{+}}\left(i x+g^{\prime}(\lambda)\right) d \lambda=\log \left(e_{+}^{2}\left(s_{j_{n}}^{+}\right) e_{-}^{2}\left(s_{k_{n}}^{-}\right)\right) .
$$




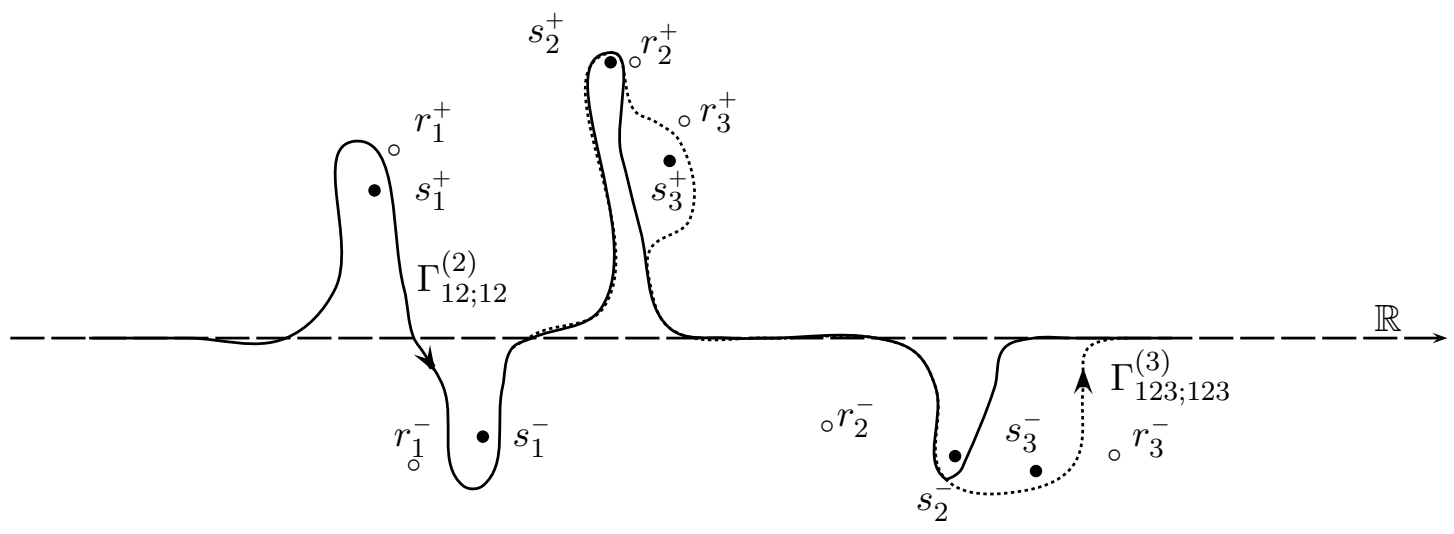

Figure 2: Integration contours $\Gamma_{12 ; 12}^{(2)}$ (solid line) and $\Gamma_{123 ; 123}^{(3)}$ (dotted line)

Let us transform now the double integral in (2.5). To lighten the notations we set

$$
\alpha\left(\lambda ; \Gamma_{J ; K}^{(n)}\right)=\alpha(\lambda ; n), \quad \alpha\left(\lambda ; \Gamma_{\hat{J} ; \hat{K}}^{(n-1)}\right)=\alpha(\lambda ; n-1) .
$$

We can present $\nu(\lambda)$ on the contour $\Gamma_{\hat{J} ; \hat{K}}^{(n-1)}$ as

$$
\nu(\lambda)=\frac{-1}{2 \pi i} \log \frac{\alpha_{-}(\lambda ; n-1)}{\alpha_{+}(\lambda ; n-1)}, \quad \lambda \in \Gamma_{\hat{J} ; \hat{K}}^{(n-1)} .
$$

Using this representation one can integrate over $\lambda$ in the double integral in (2.5)

$$
\int_{\substack{\Gamma_{\hat{J} ; \hat{K}}^{(n-1)}}} \frac{\nu(\lambda) \nu(\mu)}{\left(\lambda-\mu_{+}\right)^{2}} d \lambda d \mu=\int_{\substack{\Gamma_{\hat{J} ; \hat{K}}^{(n-1)}\\}} \nu(\mu) \cdot \log ^{\prime} \alpha_{+}(\mu ; n-1) d \mu .
$$

Substituting here $\nu(\mu)$ via (5.6) we arrive at

$$
\int_{\substack{(n-1) \\ \Gamma_{\hat{J} ; \hat{K}}}} \frac{\nu(\lambda) \nu(\mu)}{\left(\lambda-\mu_{+}\right)^{2}} d \lambda d \mu=\frac{-1}{2 \pi i} \int_{\Gamma_{\hat{J} ; \hat{K}}^{(n-1)}} \log \alpha_{-}(\mu ; n-1) \cdot \log ^{\prime} \alpha_{+}(\mu ; n-1) d \mu .
$$

Similarly we have on the contour $\Gamma_{J ; K}^{(n)}$

$$
\int_{\Gamma_{J ; K}^{(n)}} \frac{\nu(\lambda) \nu(\mu)}{\left(\lambda-\mu_{+}\right)^{2}} d \lambda d \mu=\frac{-1}{2 \pi i} \int_{\Gamma_{J ; K}^{(n)}} \log \alpha_{-}(\mu ; n) \cdot \log ^{\prime} \alpha_{+}(\mu ; n) d \mu .
$$

In order to compare (5.8) and (5.9) we first observe that

$$
\alpha_{ \pm}(\lambda ; n)=\alpha_{ \pm}(\lambda ; n-1)\left(\frac{\lambda-s_{k_{n}}^{-}}{\lambda-s_{j_{n}}^{+}}\right) .
$$


Let us introduce now $\hat{\alpha}_{ \pm}(\lambda)$ by

$$
\alpha_{-}(\lambda ; n-1)=\hat{\alpha}_{-}(\lambda)\left(\frac{\lambda-s_{j_{n}}^{+}}{\lambda-r_{j_{n}}^{+}}\right), \quad \alpha_{+}(\lambda ; n-1)=\hat{\alpha}_{+}(\lambda)\left(\frac{\lambda-r_{k_{n}}^{-}}{\lambda-s_{k_{n}}^{-}}\right)
$$

Due to (5.10) we also have

$$
\alpha_{-}(\lambda ; n)=\hat{\alpha}_{-}(\lambda)\left(\frac{\lambda-s_{k_{n}}^{-}}{\lambda-r_{j_{n}}^{+}}\right), \quad \alpha_{+}(\lambda ; n)=\hat{\alpha}_{+}(\lambda)\left(\frac{\lambda-r_{k_{n}}^{-}}{\lambda-s_{j_{n}}^{+}}\right) .
$$

It is easy to see that $\hat{\alpha}_{-}(\lambda)$ is analytical and non-vanishing to the right from $\Gamma_{\hat{J} ; \hat{K}}^{(n-1)}$ and $\Gamma_{J ; K}^{(n)}$, while $\hat{\alpha}_{+}^{-1}(\lambda)$ is analytical and non-vanishing to the left from $\Gamma_{\hat{J} ; \hat{K}}^{(n-1)}$ and $\Gamma_{J ; K}^{(n)}$. Moreover, both $\hat{\alpha}_{ \pm}(\lambda)$ are analytical and non-vanishing in the interior between $\Gamma_{\hat{J} ; \hat{K}}^{(n-1)}$ and $\Gamma_{J ; K}^{(n)}$.

Substituting (5.11), (5.12) into (5.8) and (5.9) we obtain

$$
\int_{\Gamma_{\hat{\jmath} ; \hat{K}}^{(n-1)}} \frac{\nu(\lambda) \nu(\mu)}{\left(\lambda-\mu_{+}\right)^{2}} d \lambda d \mu=\frac{-1}{2 \pi i} \int_{\Gamma_{\hat{\jmath} ; \hat{K}}^{(n-1)}} \log ^{\prime} \hat{\alpha}_{+}(\mu) \cdot \log \hat{\alpha}_{-}(\mu) d \mu+\log \left(\frac{\alpha_{-}\left(r_{k_{n}}^{-} ; n-1\right) \hat{\alpha}_{+}\left(s_{j_{n}}^{+}\right)}{\alpha_{-}\left(s_{k_{n}}^{-} ; n-1\right) \hat{\alpha}_{+}\left(r_{j_{n}}^{+}\right)}\right),
$$

and

$$
\int_{\Gamma_{J ; K}^{(n)}} \frac{\nu(\lambda) \nu(\mu)}{\left(\lambda-\mu_{+}\right)^{2}} d \lambda d \mu=\frac{-1}{2 \pi i} \int_{\Gamma_{J ; K}^{(n)}} \log ^{\prime} \hat{\alpha}_{+}(\mu) \cdot \log \hat{\alpha}_{-}(\mu) d \mu+\log \left(\frac{\alpha_{-}\left(r_{k_{n}}^{-} ; n\right) \hat{\alpha}_{+}\left(s_{k_{n}}^{-}\right)}{\alpha_{-}\left(s_{j_{n}}^{+} ; n\right) \hat{\alpha}_{+}\left(r_{j_{n}}^{+}\right)}\right) .
$$

Using that $\log \hat{\alpha}_{ \pm}(\mu)$ is holomorphic in the interior between $\Gamma_{\hat{J} ; \hat{K}}^{(n-1)}$ and $\Gamma_{J ; K}^{(n)}$ we find

$$
\left(\int_{\Gamma_{J ; K}^{(n)}}-\int_{\Gamma_{\hat{\jmath} ; \hat{K}}^{(n-1)}}\right) \frac{\nu(\lambda) \nu(\mu)}{\left(\lambda-\mu_{+}\right)^{2}} d \lambda d \mu=\log \left(\frac{\alpha_{-}\left(s_{k_{n}}^{-} ; n-1\right) \cdot \alpha_{-}\left(r_{k_{n}}^{-} ; n\right) \cdot \hat{\alpha}_{+}\left(s_{k_{n}}^{-}\right)}{\alpha_{-}\left(r_{k_{n}}^{-} ; n-1\right) \cdot \alpha_{-}\left(s_{j_{n}}^{+} ; n\right) \cdot \hat{\alpha}_{+}\left(s_{j_{n}}^{+}\right)}\right) .
$$

The last step is to express the obtained answer in terms of $\alpha_{ \pm}(\lambda ; n-1)$ via (5.10), (5.11). We will also need the following evident equations:

$$
\begin{aligned}
& \hat{\alpha}_{-}\left(s_{j_{n}}^{+}\right)=\left.\left(s_{j_{n}}^{+}-r_{j_{n}}^{+}\right) \frac{d}{d \lambda} \alpha_{-}(\lambda ; n-1)\right|_{\lambda=s_{j_{n}}^{+}}, \\
& \hat{\alpha}_{+}^{-1}\left(s_{k_{n}}^{-}\right)=\left.\left(s_{k_{n}}^{-}-r_{k_{n}}^{-}\right) \frac{d}{d \lambda} \alpha_{+}^{-1}(\lambda ; n-1)\right|_{\lambda=s_{k_{n}}^{-}},
\end{aligned}
$$

and

$$
\begin{aligned}
& \gamma F^{\prime}\left(s_{j_{n}}^{+}\right)=\left.\alpha_{+}^{-1}\left(s_{j_{n}}^{+} ; n-1\right) \cdot \frac{d}{d \lambda} \alpha_{-}(\lambda ; n-1)\right|_{\lambda=s_{j_{n}}^{+}} \\
& \gamma F^{\prime}\left(s_{k_{n}}^{-}\right)=\left.\alpha_{-}\left(s_{k_{n}}^{-} ; n-1\right) \frac{d}{d \lambda} \alpha_{+}^{-1}(\lambda ; n-1)\right|_{\lambda=s_{k_{n}}^{-}}
\end{aligned}
$$


Substituting these formulas into (5.15) we obtain after simple algebra

$$
\left(\int_{\Gamma_{J ; K}^{(n)}}-\int_{\Gamma_{\hat{J} ; \hat{K}}^{(n-1)}}\right) \frac{\nu(\lambda) \nu(\mu)}{\left(\lambda-\mu_{+}\right)^{2}} d \lambda d \mu=\log \left(\frac{\alpha_{-}^{2}\left(s_{k_{n}}^{-} ; n-1\right) \alpha_{+}^{-2}\left(s_{j_{n}}^{+} ; n-1\right)}{\left(s_{j_{n}}^{+}-s_{k_{n}}^{-}\right)^{2} \gamma^{2} F^{\prime}\left(s_{j_{n}}^{+}\right) F^{\prime}\left(s_{k_{n}}^{-}\right)}\right) .
$$

Combining this result with (5.4) we arrive at the statement of Lemma.

Corollary 5.1. Let $\alpha(\lambda)$ be defined by (2.1). Then

$$
\exp \left\{\mathcal{A}_{\Gamma_{J ; K}^{(n)}}([g],[\nu])-\mathcal{A}_{\mathbb{R}}([g],[\nu])\right\}=\left(\operatorname{det} \frac{1}{s_{j_{a}}^{+}-s_{k_{b}}^{-}}\right)^{2} \prod_{m=1}^{n}\left(\frac{\alpha_{-}\left(s_{k_{m}}^{-}\right)}{\alpha_{+}\left(s_{j_{m}}^{+}\right)}\right)^{2} \frac{e_{+}^{2}\left(s_{j_{m}}^{+}\right) e_{-}^{2}\left(s_{k_{m}}^{-}\right)}{\gamma^{2} F^{\prime}\left(s_{j_{m}}^{+}\right) F^{\prime}\left(s_{k_{m}}^{-}\right)}
$$

Proof. Applying successively Lemma 5.1 to the exponents

$$
\exp \left\{\mathcal{A}_{\Gamma_{j_{1}, \ldots, j_{m} ; k_{1}, \ldots, k_{m}}^{(m)}}([g],[\nu])-\mathcal{A}_{\Gamma_{j_{1}, \ldots, j_{m-1} ; k_{1}, \ldots, k_{m-1}}^{(m-1)}}([g],[\nu])\right\}
$$

for $m=1, \ldots, n$ we have

$$
\exp \left\{\mathcal{A}_{\Gamma_{J ; K}^{(n)}}-\mathcal{A}_{\mathbb{R}}\right\}=\prod_{m=1}^{n}\left(\frac{\alpha_{-}\left(s_{k_{m}}^{-} ; \Gamma_{j_{1}, \ldots, j_{m-1} ; k_{1}, \ldots, k_{m-1}}^{(m-1)}\right)}{\alpha_{+}\left(s_{j_{m}}^{+} ; \Gamma_{j_{1}, \ldots, j_{m-1} ; k_{1}, \ldots, k_{m-1}}^{(m-1)}\right)}\right)^{2} \frac{e_{+}^{2}\left(s_{j_{m}}^{+}\right) e_{-}^{2}\left(s_{k_{m}}^{-}\right)}{\left(s_{j_{m}}^{+}-s_{k_{m}}^{-}\right)^{2} \gamma^{2} F^{\prime}\left(s_{j_{m}}^{+}\right) F^{\prime}\left(s_{k_{m}}^{-}\right)}
$$

It follows from (5.10) that

$$
\alpha_{ \pm}\left(\lambda ; \Gamma_{j_{1}, \ldots, j_{m-1} ; k_{1}, \ldots, k_{m-1}}^{(m-1)}\right)=\alpha_{ \pm}(\lambda) \prod_{a=1}^{m-1}\left(\frac{\lambda-s_{k_{a}}^{-}}{\lambda-s_{j_{a}}^{+}}\right) .
$$

It remains to substitute this into (5.20) and to use the equation

$$
\underset{\substack{j_{1}, \ldots, j_{n} \\ k_{1}, \ldots, k_{n}}}{\operatorname{det}} \frac{1}{s_{j_{a}}^{+}-s_{k_{b}}^{-}}=\frac{\prod_{a>b}^{n}\left(s_{j_{a}}^{+}-s_{j_{b}}^{+}\right)\left(s_{k_{b}}^{-}-s_{k_{a}}^{-}\right)}{\prod_{a, b=1}^{n}\left(s_{j_{a}}^{+}-s_{k_{b}}^{-}\right)} .
$$

Proof of Theorem 2.3. The asymptotic behavior of the Fredholm determinant (4.10) contains the determinant $\operatorname{det}(I-A)$ with $A=A^{-} A^{+}$. Let for definiteness $n_{+} \leq n_{-}$(otherwise $\operatorname{det}(I-A)$ can be replaced by $\operatorname{det}(I-\tilde{A})$ with $\tilde{A}=A^{+} A^{-}$due to (4.12) $)$. Then one has

$$
\operatorname{det}_{n_{+}}(I-A)=\sum_{n=0}^{n_{+}} \sum_{j_{n}>\cdots>j_{1}}^{n_{+}} \operatorname{det}_{a, b=1, \ldots, n}\left(-\sum_{k=1}^{n_{-}} A_{j_{a}, k}^{-} A_{k, j_{b}}^{+}\right)
$$


Substituting here $A^{ \pm}$from (2.4) we obtain

$$
\operatorname{det}_{n_{+}}(I-A)=\sum_{n=0}^{n_{+}} \sum_{j_{n}>\cdots>j_{1}}^{n_{+}} \sum_{k_{1}, \ldots, k_{n}=1}^{n_{-}} \prod_{a=1}^{n} \frac{h_{k_{a}}^{-} h_{j_{a}}^{+} e_{-}^{2}\left(s_{k_{a}}^{-}\right) e_{+}^{2}\left(s_{j_{a}}^{+}\right)}{s_{j_{a}}^{+}-s_{k_{a}}^{-}} \operatorname{det}_{a, b=1, \ldots, n}\left(\frac{1}{s_{j_{a}}^{+}-s_{k_{b}}^{-}}\right)
$$

It remains to use the explicit expressions (2.4) for $h^{ \pm}$and to make the replacement

$$
\prod_{a=1}^{n}\left(s_{j_{a}}^{+}-s_{k_{a}}^{-}\right)^{-1} \mapsto \frac{1}{n !} \operatorname{det}_{a, b=1, \ldots, n}\left(\frac{1}{s_{j_{a}}^{+}-s_{k_{b}}^{-}}\right) .
$$

Such the replacement is possible, since we take the sum over all $j_{\ell}$ and $k_{\ell}$. Then due to (5.19) we arrive at

$$
\operatorname{det}_{n_{+}}(I-A)=\sum_{n=0}^{n_{+}} \sum_{j_{n}>\cdots>j_{1}}^{n_{+}} \sum_{k_{n}>\cdots>k_{1}}^{n_{-}} \exp \left\{\mathcal{A}_{\Gamma_{J ; K}^{(n)}}([g],[\nu])-\mathcal{A}_{\mathbb{R}}([g],[\nu])\right\} .
$$

Taking into account (2.10) we obtain the statement of Theorem.

\section{$6 \quad$ Examples}

In this section we consider applications of the results obtained.

\subsection{Temperature correlation function of impenetrable bosons}

The operator of number of particles $Q_{x}$ on an interval $[0, x]$ plays an important role in the theory of quantum one-dimensional integrable systems [29]-[31]. The expectation value of $e^{\beta Q_{x}}$, where $\beta$ is a complex parameter, is a generating function for some correlation functions of such models. In the model of impenetrable bosons at finite temperature this expectation value is given by the Fredholm determinant of the operator $I+\gamma V$ (1.2) with $g(\lambda)=0$ and

$$
\gamma F(\lambda)=\frac{e^{\beta}-1}{e^{\frac{\lambda^{2}-h}{T}}+1}
$$

where $h$ is the chemical potential and $T$ is the temperature. The leading terms of the large $x$ asymptotic expansion of this determinant were calculated in [18, 19, 31]. The formulas (2.10), (2.11) give the complete asymptotic expansion. Hereby

$$
\nu(\lambda)=\frac{-1}{2 \pi i} \log \left(\frac{e^{\frac{\lambda^{2}-h}{T}}+e^{\beta}}{e^{\frac{\lambda^{2}-h}{T}}+1}\right),
$$

and the function $\alpha(\lambda)$ is given by (2.1). The roots of the equation $1+\gamma F(\lambda)=0$ form two series $s_{j, i}^{ \pm}, i=1,2$ :

$$
s_{j, 1}^{+}=\sqrt{h+\beta T+i \pi T(2 j+1)}, \quad j=0,1, \ldots,
$$


and $s_{j, 2}^{+}=-\left(s_{j, 1}^{+}\right)^{*}, s_{j, 1}^{-}=\left(s_{j, 1}^{+}\right)^{*}, s_{j, 2}^{-}=-\left(s_{j, 1}^{-}\right)^{*}$. The equations (2.10), (2.11) are valid for arbitrary $N=n_{+}=n_{-}$. For $N$ fixed we neglect the roots $s_{N+1}^{ \pm}$, whose contribution is of order $e^{i x\left(s_{N+1, j}^{+}-s_{1, k}^{-}\right)}$. Therefore one can set $a=\Im\left(s_{N+1,1}^{+}-s_{1,1}^{-}\right)$in the reminder $O\left(e^{-a x}\right)$.

\subsection{Normalization factor in the $X X Z$ spin chain}

Our second example is related to the $X X Z$ spin- $1 / 2$ Heisenberg chain in an external magnetic field. In the thermodynamic limit correlation functions of this model contain a normalization factor proportional to the Fredholm determinant $\operatorname{det}_{[-x / 2, x / 2]}(I+K)$ [32], where the operator $K\left(t-t^{\prime}\right)$ acts on the interval $[-x / 2, x / 2]$ and has the kernel

$$
K\left(t-t^{\prime}\right)=\frac{\sin 2 \zeta}{2 \pi \sinh \left(t-t^{\prime}-i \zeta\right) \sinh \left(t-t^{\prime}+i \zeta\right)}
$$

Here $0<\zeta<\pi$ and $\cos \zeta=\Delta$, where $\Delta$ is the anisotropy parameter of the model. The length of the interval $x$ depends on the value of the external magnetic field. If the last one goes to zero, then $x \rightarrow \infty$.

The operator $I+K$ belongs to the class of truncated Wiener-Hopf operators. They can be reduced to the operators with the GSK by the Fourier transform. Let $\chi_{\left[-\frac{x}{2}, \frac{x}{2}\right]}\left(t^{\prime}\right)$ be the characteristic function of the interval $[-x / 2, x / 2]$. Then

$$
\operatorname{det}_{\left[-\frac{x}{2}, \frac{x}{2}\right]}(I+K)=\operatorname{det}_{\mathbb{R}}(I+V)
$$

where the operator $I+V$ acts on $\mathbb{R}$ and

$$
V(\lambda, \mu)=\frac{1}{2 \pi} \int_{\mathbb{R}} e^{i t \lambda} K\left(t-t^{\prime}\right) \chi_{[-x / 2, x / 2]}\left(t^{\prime}\right) e^{-i t^{\prime} \mu} d t d t^{\prime} .
$$

Calculating the integral in (6.6) we find

$$
V(\lambda, \mu)=F(\lambda) \frac{\sin \frac{x}{2}(\lambda-\mu)}{\pi(\lambda-\mu)},
$$

where

$$
F(\lambda)=\hat{K}(\lambda)=\frac{\sinh [\lambda(\pi / 2-\zeta)]}{\sinh [\lambda \pi / 2]} .
$$

Thus, up to the similarity transformation we obtain the GSK with $g(\lambda)=0$ and $F(\lambda)$ given by (6.8). Hence, if $x$ is large enough (what corresponds to a small magnetic field), we can calculate the original determinant $\operatorname{det}_{[-x / 2, x / 2]}(I+K)$ asymptotically.

Just like in the previous example the formulas (2.10), (2.11) give the complete asymptotic expansion. We have

$$
\nu(\lambda)=\frac{-1}{2 \pi i} \log \left(\frac{2 \sinh [\lambda(\pi-\zeta) / 2] \cosh [\lambda \zeta / 2]}{\sinh [\lambda \pi / 2]}\right)
$$


The function $\alpha(\lambda)$ can be found explicitly in terms of $\Gamma$-functions

$$
\alpha_{-}(\lambda)=\sqrt{2(\pi-\zeta)}\left(\frac{\pi}{\zeta}\right)^{-\frac{i \lambda \zeta}{2 \pi}}\left(\frac{\pi}{\pi-\zeta}\right)^{-\frac{i \lambda(\pi-\zeta)}{2 \pi}} \frac{\Gamma\left(1+\frac{i \lambda}{2}\right)}{\Gamma\left(\frac{1}{2}+\frac{i \lambda \zeta}{2 \pi}\right) \Gamma\left(1+\frac{i \lambda(\pi-\zeta)}{2 \pi}\right)},
$$

and $\alpha_{+}^{-1}(\lambda)=\alpha_{-}(-\lambda)$. Similarly to the case considered above the roots of the equation $1+$ $\gamma F(\lambda)=0$ form two series $s_{j, i}^{ \pm}, i=1,2$. If $\pi / \zeta$ is not a rational number, then

$$
s_{j, 1}^{+}=\frac{2 \pi i}{\pi-\zeta}(j+1), \quad s_{j, 2}^{+}=\frac{\pi i}{\zeta}(2 j+1), \quad j=0,1, \ldots
$$

and $s_{j, i}^{-}=\left(s_{j, i}^{+}\right)^{*}$. If $\pi / \zeta$ is a rational number, then one should omit in (6.11) such $s_{j, i}^{ \pm}$that satisfy the condition $\sinh \left(\pi s_{j, i}^{ \pm} / 2\right)=0$. The asymptotic estimates (2.10), (2.11) are valid for arbitrary $N=n_{+}=n_{-}$. Similarly to the case considered above one can set $a=\min _{i, i^{\prime}=1,2} \Im\left(s_{N+1, i}^{+}-s_{1, i^{\prime}}^{-}\right)$ in the reminder is $O\left(e^{-a x}\right)$.

\section{Acknowledgements}

I am grateful to N. Kitanine, K.K. Kozlowski, J.M. Maillet and V. Terras for useful discussions. This work was supported in parts by French-Russian network in Theoretical and Mathematical Physics (GDRI-471 of CNRS and RFBR-CNRS-09-01-93106L-a), the Program of RAS "Mathematical Methods of the Nonlinear Dynamics", RFBR-08-01-00501a, RFBR-09-01-12150ofi-m, NS-8265.2010.1.

\section{References}

[1] M. Gaudin, Nucl. Phys. 25 (1961), 447.

[2] M. Gaudin and M. L. Mehta, Nucl. Phys 18 (1960), 420.

[3] A. Lenard, J. Math. Phys. 5 (1964), 930.

[4] M. Jimbo, T. Miwa, Y. Mori, and M. Sato, Physica D 1 (1980), 80.

[5] J. des Cloizeaux and M. L. Mehta, J. Math. Phys. 14 (1973), 1648.

[6] H. Widom, Indiana Univ. Math. J. 21 (1971), 277.

[7] F. Dyson, Comm. Math. Phys. 47 (1976), 171.

[8] H. Widom, J. Approx. th. 77 (1994), 51.

[9] H. Widom, Comm. Math. Phys. 171 (1995), 159. 
[10] V. I. Krasovsky, Int. Math. Res. Not. 2004 (2004), 1249-1272.

[11] T. Ehrhardt, Comm. Math. Phys. 262 (2006), 317.

[12] P. A. Deift, A. R. Its, I. Krasovsky and X. Zhou, J. Comput. Appl. Math. 202 (2007), no. $1,26$.

[13] P. A. Deift, A.R. Its and X. Zhou, Ann. Math. 146 (1997), 149.

[14] V. A. Fock, Mat. Sborn. 56 (1944), 3.

[15] B. Noble, Methods based on the Wiener-Hopf technique for the solution of partial differential equations, Pergamon Press (1958)

[16] B. M. McCoy, J. H. H. Perk, and R. E. Shrock, Nucl. Phys. B 220 (1983), 35.

[17] A. Lenard, J. Math. Phys. 7 (1966), 1268.

[18] A.R. Its, A.G. Izergin, and V.E. Korepin, Commun. Math. Phys. 129 (1990), 205.

[19] A.R. Its, A.G. Izergin, and V.E. Korepin, Commun. Math. Phys. 130 (1990), 471.

[20] F. Colomo, A. G. Izergin, V. E. Korepin, and V. Tognetti, Phys. Lett. A 169 (1992), 237.

[21] F. Colomo, A. G. Izergin, V. E. Korepin, and V. Tognetti, Theor. Mat. Phys. 94 (1993), 11.

[22] A.R. Its, A.G. Izergin, V.E. Korepin, and N. A. Slavnov, Phys. Rev. Lett. 70 (1993), 1704.

[23] A.R. Its, A.G. Izergin, V.E. Korepin, and N.A. Slavonv, Int. J. Mod. Physics B4 (1990), 1003.

[24] V.V. Cheianov and M.R. Zvonarev, J. Phys. A:Math. Gen., 37 (2004), 2261.

[25] N. Kitanine, K. K. Kozlowski, J.-M. Maillet, N.A. Slavnov, and V. Terras, Commun. Math. Phys. 291 (2009) 691.

[26] A. M. Budylin and V. S. Buslaev, Algebra i Analiz no 67 (1995), 925.

[27] P. Deift, In: Differential operators and spectral theory: M. Sh. Birman's 70th anniversary collection (V. Buslaev, M. Solomyak, D. Yafaev, eds.), American Mathematical Society Translations, ser. 2, 189, Providence, R.I.: AMS, (1999), 69.

[28] N. I. Akhiezer, Ukrainian Math. J. 16 (1964), 445.

[29] A.G. Izergin, V.E. Korepin, Comunn. Math. Phys. 94 (1984), 67.

[30] A.G. Izergin, V.E. Korepin, Comunn. Math. Phys. 99 (1985), 271. 
[31] V. E. Korepin, N. M. Bogoliubov, A. G. Izergin, Quantum Inverse Scattering Method and Correlation Functions, Cambridge University Press, 1993.

[32] V.E. Korepin, Comunn. Math. Phys. 86 (1984), 391. 